\title{
Audit of the Diagnosis and Management of Hypogonadism in Adult Men
}

P W James Russell1', Javier Gomez², K Swe Myint1

1. Elsie Bertram Diabetes Centre, Norfolk and Norwich University Hospital, Norwich Research Park, United Kingdom, NR4 7UY

2. Clinical Biochemistry Department, Norfolk and Norwich University Hospital, Norwich Research Park, United Kingdom, NR4 7UY

\section{Background}

Symptomatic hypogonadism affects $5-6 \%$ of men aged $30-79$ years and is associated with increased morbidity and mortality. ${ }^{1}$ There are currently no trust guidelines for the diagnostic workup of these patients.

\section{Methods}

Retrospective analysis of the diagnosis and assessment of adult men with low serum total testosterone (TT) and compared with the clinical practice guidelines of The Endocrine Society. ${ }^{2}$

We identified all patients with TT below normal range $(<9.9 \mathrm{nmol} / \mathrm{L})$ carried out at the trust between July and November 2010 and excluded patients with known hypogonadism or prostate cancer.

First 50 cases were evaluated in detail using electronic records and clinic letters.

\section{Diagnosis of Hypogonadism}

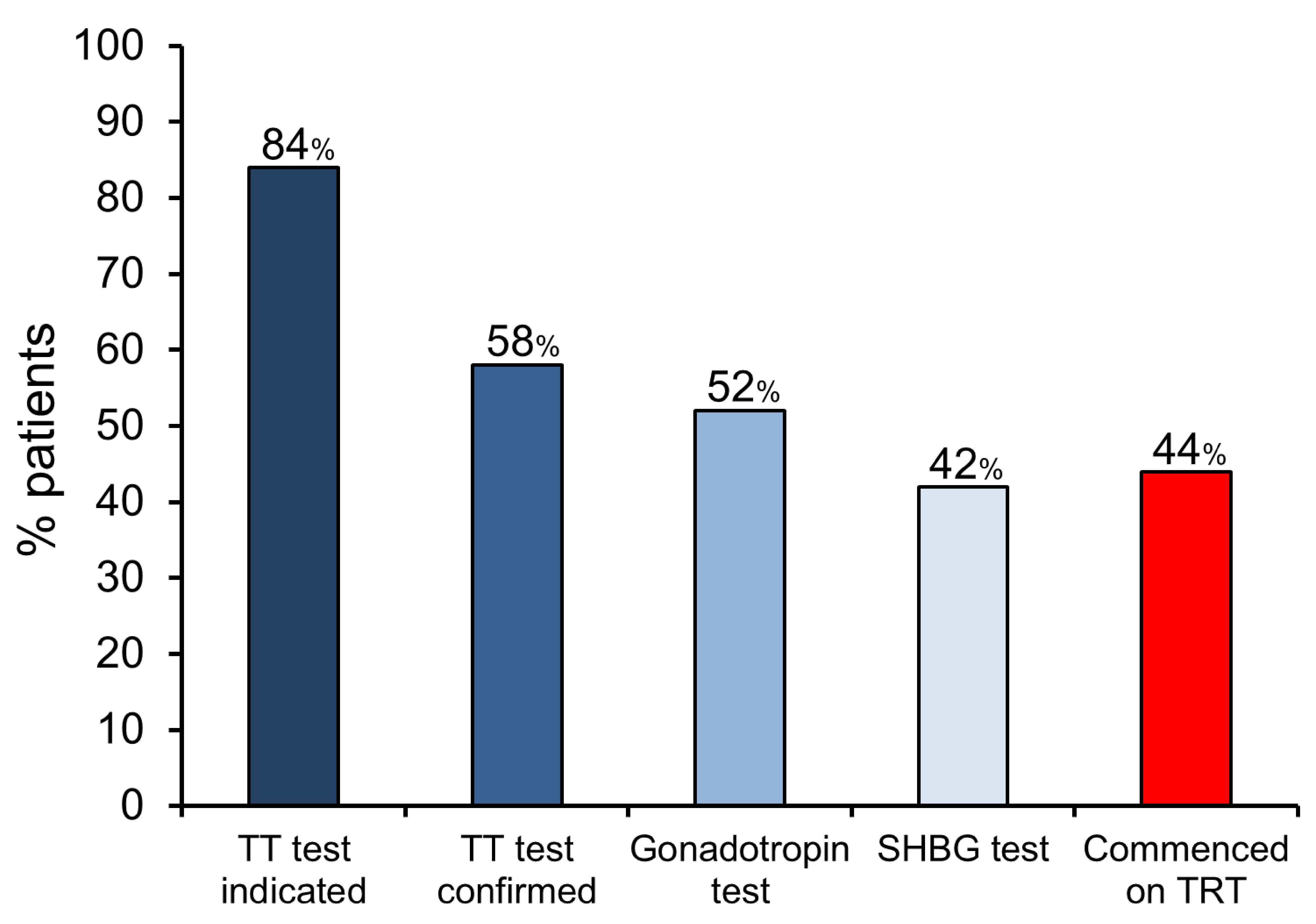

Figure 1: Diagnostic Testing of Suspected Hypogonadism.

In total, 247 cases with low TT were identified. Among 50 patients (mean age 63, range 34-94 years) the mean time at which TT was tested was 11:18am. \pm 142 minutes.

\section{Management of Testosterone Therapy}

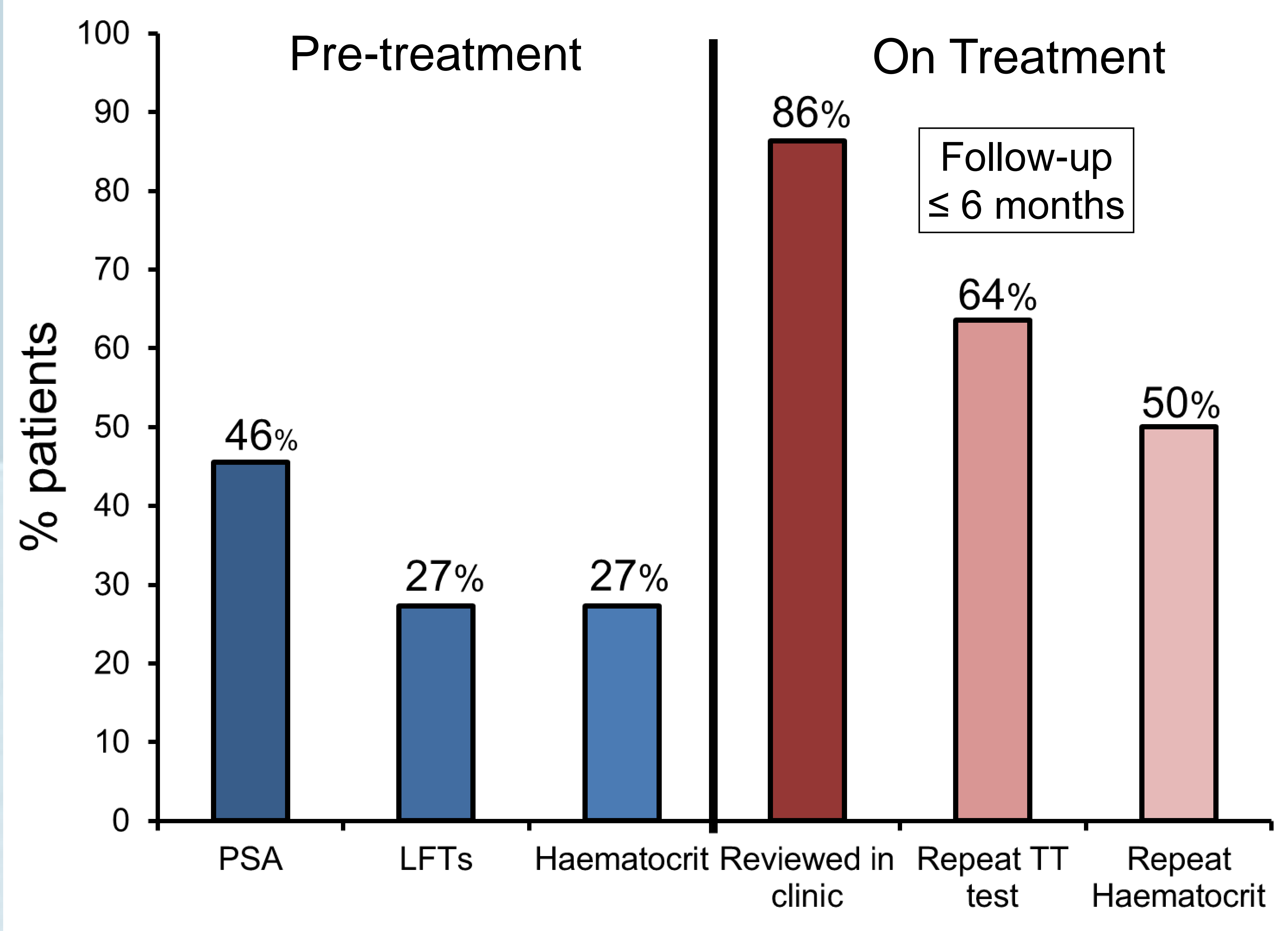

Figure 2: Pre-treatment Assessment and Follow-up of Therapy.

Gonadotropins were tested in $52 \%$ of patients, of which $69.2 \%$ were low/normal and $26.9 \%$ were high. Of those with low/normal levels, $50 \%$ had complete pituitary function testing (cortisol, prolactin, free thyroxine).

\section{Conclusions}

There are pitfalls in our practice in both diagnosis and assessment of hypogonadism. Morning TT is measured later than recommended and often not repeated, resulting in potential over-diagnosis. Lack of gonadotropin measurement has serious implications for missing potential aetiology. Pre-treatment assessment and treatment monitoring must be improved to ensure patient safety. We are currently writing trust guidelines to address these issues.

\section{References}

Araujo, A. B. et al. Prevalence and incidence of androgen deficiency in middle-aged and older men: estimates from the Massachusetts Male Aging Study. The Journal of clinical endocrinology and metabolism 89, 5920-6 (2004)

2. Bhasin, S. et al. Testosterone therapy in men with androgen deficiency syndromes: an Endocrine Society clinical practice guideline. The Journal of clinical endocrinology and metabolism 95, 2536-59 (2010) 